\title{
Polarization observables and spin-dependent distortion effects in single-nucleon knockout reactions
}

\author{
A. García-Camacho, ${ }^{*}$ R. C. Johnson, and J. A. Tostevin \\ Department of Physics, School of Electronics and Physical Sciences, University of Surrey, Guildford, Surrey GU2 7XH, United Kingdom
}

(Received 27 May 2005; published 19 October 2005)

\begin{abstract}
A formalism is developed and predictions are made for selected polarization observables in one-nucleon knockout reactions within the transfer-to-the-continuum direct reaction model. The effects of nucleon-target spin-orbit distortions are also included and their importance is quantified. The calculated knockout reaction analyzing powers reach an appreciable fraction of their maximum allowed values. They are also found to be quite sensitive to the different approximation schemes used for the transfer probability amplitude within the transfer-to-the-continuum model. In contrast, they are shown to be insensitive to details of the two-body interactions assumed, including the nucleon-target spin-orbit interaction. The angular distributions of $\gamma$-rays, arising from the decay of heavy reaction residues produced in excited final states, are shown to be strongly anisotropic.
\end{abstract}

DOI: 10.1103/PhysRevC.72.044603

PACS number(s): 21.10.Jx, 24.10.-i, 24.70.+s, 27.30.+t

\section{INTRODUCTION}

The use of nucleon knockout reaction partial cross sections, and their associated residue momentum distributions, for the single particle spectroscopy of light exotic nuclei has been the subject of recent studies and reviews [1-4]. Several direct reaction models have also been developed to analyze and interpret different aspects of these experimental data. Among these is the transfer-to-the-continuum (TC) direct reaction model, as developed by Bonaccorso and Brink [5].

The potential to exploit spin degrees of freedom and polarization observables could significantly enhance and supplement this spectroscopic role, as well as elucidating studies of the reaction dynamics and the reaction mechanism. In particular, the angular distributions of the $\gamma$-rays emitted by the heavy residue following the nucleon removal process are becoming amenable to experimental study [6]. We also require a better knowledge of the secondary beam polarizations expected from the fragmentation process itself [7-9]. When discussing such polarization phenomena, the possible role of spin-dependent distortions from the interactions between the projectile's constituents and the target must also be considered. This spin-dependence (the spin-orbit force) in the nucleon-target interaction can be readily included within the TC approach adopted here. This is not the case in the much-used eikonal approaches (see, for example Ref. [10] for the deuteron case) where a first step is the use of an adiabatic or sudden approximation [11] for the internal motion of the projectile.

In this work we therefore generalize the TC model for the study of polarization and nucleon-target spin-orbit distortion effects in single nucleon knockout reactions. Spin-orbit interaction effects have been included previously, both in the

\footnotetext{
*Electronic address: camacho@df.unipi.it; Present address: Instituto Nazionale di Fisica Nucleare, Sezione di Pisa, I-56125 Pisa, Italy.
}

semiclassical formulation of transfer reactions between bound states of heavy ions $[12,13]$, where they affect selectivity with respect to the bound state angular momentum quantum numbers, and, as a natural extension, to the TC model of nuclear breakup reactions $[14,20,23]$ as a spin-orbit distortion. In the former case [13], this distortion was essential in the reproduction of the position of the target single particle resonances, whereas in the latter [20,23] spin-orbit effects were shown to be important to determine the spin value of the single particle state from which breakup originates. However, these were always studied in conjunction with further approximations to the TC reaction amplitude. Here, these nucleon-target spin-orbit distortion effects are studied with and without such additional approximations [15]. Regarding studies of analyzing powers, in Ref. [16] the TC formalism was applied in calculations of transfer reactions between heavy nuclei. One of our aims is to assess this approach in applications to one-nucleon knockout from lighter and also more weakly-bound nuclei.

In Sec. II the main formalism is developed and the basis of the TC model is discussed. The formalism for analyzing powers is also presented. In Sec. III the reactions under study and the two-body interactions that we use are described. Calculations of polarized momentum distributions, analyzing powers and $\gamma$-ray angular distributions are then presented. Section IV discusses our choice of the nucleon-target spinorbit interaction. Finally, in Sec. V, the spin-orbit distortion effects are included and their importance for the calculated observables is discussed.

\section{FORMALISM}

The transfer-to-the-continuum (TC) model has evolved from theories of the (one-step) transfer of a nucleon (or cluster of nucleons) between bound states in heavy nuclei. The essential step in these earlier bound states formulations involved making a semiclassical approximation to the entrance and exit channel distorted waves in the distorted-waves 
Born approximation (DWBA) model of transfer reactions [12,13,17-19]. These bound state models were then generalized and applied to treat, approximately, nucleon/cluster transfers to both resonant and nonresonant final states in the continuum - thus the projectile breakup is viewed as a particle transfer into continuum final states of the nucleon and target system $[5,20]$. It is therefore assumed that the nucleontarget interaction is the dominant interaction in the final state.

The TC formulation also assumes that the nucleon-core potential, that initially binds the projectile, and the nucleon-target potential, responsible for the breakup of the projectile, have no spatial overlap during the entire collision. The TC model is therefore most applicable to weakly bound systems and to neutron knockout or transfer processes. This nonoverlapping potential approximation allows the use of external forms for both the nucleon-core and the nucleon-target wave functions [20]. Additionally, in the TC model, the core-target motion is treated semiclassically while the neutron-target interaction is treated quantum mechanically $[5,17,20]$. Here, we adopt the notation developed in Ref. [15].

\section{A. Cross section}

The projectile initial state wave function is described as a bound state of a neutron and a core $c$. The projectile beam direction is chosen to be the coordinate $z$-axis. The single-particle wave function has total angular momentum $j_{1}$, with projection $n_{1}$, orbital angular momentum $l_{1}$ and an external wave function normalisation constant $C_{i}$. The probability amplitude for the reaction in which the neutron with final spin projection $\sigma$ is transferred out of the projectile is [15]

$$
\begin{aligned}
T\left(j_{1} n_{1}, \sigma \boldsymbol{k}_{f} \boldsymbol{K}\right) \\
=\frac{16 \pi^{2} \hbar C_{i}}{m v K k_{f}} \sum_{j_{2} n_{2} m_{1} m_{2} \sigma^{\prime} \lambda L l_{2}} i^{m_{1}-m_{2}} \hat{L} \exp \left(2 i \delta_{L}\right)\left(l_{2} \lambda s \sigma \mid j_{2} n_{2}\right) \\
\quad \times\left(l_{1} m_{1} s \sigma^{\prime} \mid j_{1} n_{1}\right)\left(l_{2} m_{2} s \sigma^{\prime} \mid j_{2} n_{2}\right) Y_{L m_{1}-m_{2}}(\widehat{K}) Y_{l_{2} \lambda}\left(\widehat{k}_{f}\right) \\
\quad \times K_{m_{1}-m_{2}}(\eta b) \mathcal{J}_{l_{2} j_{2}} Y_{l_{1} m_{1}}\left(\beta_{1}, \pi\right) Y_{l_{2} m_{2}}^{*}\left(\beta_{2}, 0\right)
\end{aligned}
$$

where we use the notation $\hat{L}=\sqrt{2 L+1}$ and where

$$
\mathcal{J}_{l_{2} j_{2}}=\left[1-S_{l_{2} j_{2}}\right] / 2 \text {. }
$$

Here $S_{l_{2} j_{2}}$ is the neutron-target elastic $S$-matrix at energy $\hbar^{2} k_{f}^{2} / 2 m$ where $\boldsymbol{k}_{f}$ is the final state neutron-target relative motion wave vector. $j_{2}$ and $l_{2}$ are the final state neutron-target total and orbital angular momenta and $s=1 / 2$ is the intrinsic spin of the neutron. The core-target relative motion wave vector is denoted by $\boldsymbol{K}$. The core-target scattering is described by the partial wave phase shifts $\delta_{L}$ where $L$ labels the core-target relative orbital angular momentum. The projectile bound state asymptotic normalisation constant $C_{i}$ and the dynamical model parameters $\eta, \beta_{1}$, and $\beta_{2}$ are defined by Eqs. (17), (21), and (22) of Ref. [15].

When the intrinsic angular momentum of the core, $I_{c}$, with projection $\xi_{c}$, is included the resulting probability amplitude is

$$
\begin{aligned}
T\left(j_{1}\right. & \left.n_{1}, \sigma \xi_{c} \boldsymbol{k}_{f} \boldsymbol{K}\right)=\frac{16 \pi^{2} \hbar C_{i}}{m v K k_{f}} \\
& \times \sum_{j_{2} l_{2} n_{2} m_{1} m_{2} \sigma^{\prime} \lambda L \xi_{n}} i^{m_{1}-m_{2}} \hat{L} \exp \left(2 i \delta_{L}\right)\left(l_{2} \lambda s \sigma \mid j_{2} n_{2}\right) \\
& \times\left(l_{1} m_{1} s \sigma^{\prime} \mid I_{n} \xi_{n}\right)\left(I_{n} \xi_{n} I_{c} \xi_{c} \mid j_{1} n_{1}\right)\left(l_{2} m_{2} s \sigma^{\prime} \mid j_{2} n_{2}\right) \mathcal{J}_{l_{2} j_{2}} \\
& \times Y_{L m_{1}-m_{2}}(\widehat{K}) Y_{l_{2} \lambda}\left(\widehat{k}_{f}\right) K_{m_{1}-m_{2}}(\eta b) \\
& \times Y_{l_{1} m_{1}}\left(\beta_{1}, \pi\right) Y_{l_{2} m_{2}}^{*}\left(\beta_{2}, 0\right),
\end{aligned}
$$

where the angular momentum coupling order is assumed to be $\left[\left[l_{1} \otimes s\right] I_{n} \otimes I_{c}\right] j_{1}$. The corresponding expression for the momentum distribution of the heavy residues following onenucleon removal is given by

$$
\begin{aligned}
\frac{d \sigma}{d k_{1}}= & 32 \pi\left|C_{i}\right|^{2} \frac{\mu_{i}}{\mu_{f}} \frac{\hbar}{m v k_{f}} \\
& \times \int d \boldsymbol{b} P_{e l}(b) \sum_{j_{2} l_{2} m_{1} m_{1}^{\prime} m_{2} m_{2}^{\prime}} \delta_{m_{1}-m_{1}^{\prime}, m_{2}-m_{2}^{\prime}}\left|K_{m_{1}-m_{2}}(\eta b)\right|^{2} \\
& \times(-1)^{m_{2}^{\prime}-m_{2}}\left|\mathcal{J}_{l_{2} j_{2}}\right|^{2}\left[Y_{l_{1} m_{1}}\left(\beta_{1}, 0\right) Y_{l_{1} m_{1}^{\prime}}^{*}\left(\beta_{1}, 0\right)\right] \\
& \times\left[Y_{l_{2} m_{2}}^{*}\left(\beta_{2}, 0\right) Y_{l_{2} m_{2}^{\prime}}\left(\beta_{2}, 0\right)\right] \\
& \times \frac{\hat{j}_{2}^{2}}{\hat{l}_{2} \hat{l}_{1}}(-1)^{2 s+j_{2}-l_{1}-l_{2}+I_{n}} \sum_{r \rho} \hat{r}^{2}\left(l_{1} m_{1} r \rho \mid l_{1} m_{1}^{\prime}\right) \\
& \times\left(l_{2} m_{2} r \rho \mid l_{2} m_{2}^{\prime}\right) W\left(l_{2} s l_{2} s ; j_{2} r\right) W\left(l_{1} s l_{1} s ; I_{n} r\right)
\end{aligned}
$$

where the sum over core-target partial waves $L$ has been transformed into an integral over their impact parameter $b$. Here $\hbar k_{1}$ is the $(z-)$ component of the neutron's momentum parallel to the beam direction in the projectile's rest frame. In this frame the core's parallel momentum is $-\hbar k_{1}$ which must then be boosted into the laboratory frame to make comparisons with the experimentally measured residue cross section parallel momentum distribution, $d \sigma / d p_{\|}$.

In the absence of a neutron-target spin-orbit interaction the sum on $j_{2}$ can be carried out and Eq. (4) reduces to

$$
\begin{aligned}
\frac{d \sigma}{d k_{1}}= & \frac{32 \pi\left|C_{i}\right|^{2}}{\left(2 l_{1}+1\right)} \frac{\mu_{i}}{\mu_{f}} \frac{\hbar}{m v k_{f}} \int d \boldsymbol{b} P_{e l}(b) \\
& \times \sum_{l_{2} m_{1} m_{2}}\left|K_{m_{1}-m_{2}}(\eta b)\right|^{2}\left|\mathcal{J}_{l_{2}}\right|^{2}\left|Y_{l_{1} m_{1}}\left(\beta_{1}, 0\right) Y_{l_{2} m_{2}}^{*}\left(\beta_{2}, 0\right)\right|^{2} .
\end{aligned}
$$

In all cases above, the cross section from the inelastic breakup mechanism, also referred to as the stripping mechanism, which takes into account all inelastic excitations of the target, can also be included by redefining [5]

$$
\left|\mathcal{J}_{l_{2} j_{2}}\right|^{2}=\left[\left\{1-\left|S_{l_{2} j_{2}}\right|^{2}\right\}+\left|1-S_{l_{2} j_{2}}\right|^{2}\right] / 4 \text {. }
$$

The elastic and inelastic breakup terms are now represented by $\left|1-S_{l_{2} j_{2}}\right|^{2}$ and $1-\left|S_{l_{2} j_{2}}\right|^{2}$, respectively.

The aims of this paper are now threefold, (i) to derive formulas for the reaction analyzing powers from the probability amplitude, Eq. (1), in cases where the core state spin is not observed, (ii) to obtain final-state core sub-state populations from Eq. (3), and (iii) to assess the importance of the neutron-target spin-orbit distortion effects by comparing the predictions of Eqs. (5) and (4). 


\section{B. Spin observables}

In order to calculate analyzing powers for knockout, we must construct the associated reaction spin-efficiency matrix $R_{n_{1} n_{1}^{\prime}}$, defined by

$$
R_{n_{1} n_{1}^{\prime}}=k_{f}^{2} \int d \widehat{k}_{f} d \widehat{K} \sum_{\sigma} T\left(j_{1} n_{1}, \sigma \boldsymbol{k}_{f} \boldsymbol{K}\right)\left[T\left(j_{1} n_{1}^{\prime}, \sigma \boldsymbol{k}_{f} \boldsymbol{K}\right)\right]^{*} .
$$

Here the probability amplitude $T$ is given by Eq. (1) and $d \widehat{k}_{f}$ denotes integration over only the directions of $\boldsymbol{k}_{f}$. This expression is valid in an experiment where no measurements are made of the directions of the core or neutron final state momenta. When analyzing powers for the integrated cross section are discussed in Sec. III C, then the magnitude of $k_{f}$ is also integrated over in Eq. (7).

Knowledge of this efficiency matrix allows predictions to be made for a knockout experiment with an incident projectile beam in an arbitrary spin state, involving both diagonal and off-diagonal density matrix elements, provided that the spin projection $\sigma$ of the knocked out neutron is not observed. This condition leads to the incoherent summation over $\sigma$ in Eq. (7).

The efficiency matrix is usually parametrized in terms of a set of analyzing powers $T_{k q}, k=0,1, \ldots 2 j_{1},|q| \leqslant k$, defined by

$$
T_{k q}=N_{k q} / N_{00},
$$

where

$$
N_{k q}=\sum_{n_{1}, n_{1}^{\prime}} \hat{k}\left(j_{1} n_{1} k q \mid j_{1} n_{1}^{\prime}\right) R_{n_{1} n_{1}^{\prime}} .
$$

When the amplitude, Eq. (1), is substituted in Eq. (7)

$$
\begin{aligned}
R_{n_{1} n_{1}^{\prime}}= & \int d \boldsymbol{b} P_{e l}(b) \\
& \times \sum_{m_{1}^{\prime} m_{2}^{\prime} j_{2} l_{2} n_{2} m_{1} m_{2} \sigma^{\prime} \sigma^{\prime \prime}} \delta_{m_{1}-m_{1}^{\prime}, m_{2}-m_{2}^{\prime}}\left(l_{1} m_{1} s \sigma^{\prime} \mid j_{1} n_{1}\right) \\
& \times\left(l_{1} m_{1}^{\prime} s \sigma^{\prime \prime} \mid j_{1} n_{1}^{\prime}\right)\left(l_{2} m_{2} s \sigma^{\prime} \mid j_{2} n_{2}\right)\left(l_{2} m_{2}^{\prime} s \sigma^{\prime \prime} \mid j_{2} n_{2}\right) \\
& \times\left|\mathcal{J}_{l_{2} j_{2}}\right|^{2}\left|K_{m_{1}-m_{2}}(\eta b)\right|^{2}(-1)^{m_{2}^{\prime}-m_{2}}\left[Y_{l_{1} m_{1}}\left(\beta_{1}, 0\right)\right. \\
& \left.\times Y_{l_{1} m_{1}^{\prime}}^{*}\left(\beta_{1}, 0\right)\right]\left[Y_{l_{2} m_{2}}^{*}\left(\beta_{2}, 0\right) Y_{l_{2} m_{2}^{\prime}}\left(\beta_{2}, 0\right)\right],
\end{aligned}
$$

where, as we will take ratios, overall multiplicative factors resulting from the integral have been suppressed. The numerator, $N_{k q}$, of the analyzing power of rank $k$ and projection $q$ can be evaluated in the more explicit form [22]

$$
\begin{aligned}
N_{k q}= & \int d \boldsymbol{b} P_{e l}(b) \sum_{m_{1}^{\prime} m_{2}^{\prime} j_{2} l_{2} m_{1} m_{2}} \delta_{m_{1}-m_{1}^{\prime}, m_{2}-m_{2}^{\prime}}\left|K_{m_{1}-m_{2}}(\eta b)\right|^{2} \\
& \times\left[Y_{l_{1} m_{1}}\left(\beta_{1}, 0\right) Y_{l_{1} m_{1}^{\prime}}^{*}\left(\beta_{1}, 0\right)\right] \hat{j}_{2}^{2}\left|\mathcal{J}_{l_{2} j_{2}}\right|^{2}\left[Y_{l_{2} m_{2}}^{*}\left(\beta_{2}, 0\right)\right. \\
& \left.\times Y_{l_{2} m_{2}^{\prime}}\left(\beta_{2}, 0\right)\right] \sum_{r \rho h \eta}(-1)^{G}\left(l_{1} m_{1}^{\prime} l_{2}-m_{2}^{\prime} \mid r-\rho\right)[\hat{r} \hat{h} / \hat{k}] \\
& \times\left(r-\rho l_{1}-m_{1} \mid h \eta\right)\left(h \eta l_{2} m_{2} \mid k q\right) \\
& \times W\left(l_{1} l_{2} j_{1} j_{2} ; r s\right)\left\{\begin{array}{ccc}
l_{2} & k & h \\
j_{2} & j_{1} & r \\
s & j_{1} & l_{1}
\end{array}\right\}
\end{aligned}
$$

where $G$ is the phase factor $G=l_{2}-m_{2}+l_{1}-m_{1}-k-$ $q-2 s$.

The Kronecker delta functions in Eqs. (10) and (11) [and also Eq. (4)] arise from the integration over $\widehat{K}$ in Eq. (7). This, together with the integration over $\widehat{k}_{f}$, have as a consequence that the $T_{k q}$ vanish unless $q=0$. It can also be shown that $k$ must be even. These two results are general properties of the analyzing powers of this type of experiment.

If the nucleon-target spin-orbit force can be neglected, so that $\mathcal{J}_{l_{2} j_{2}} \simeq \mathcal{J}_{l_{2}}$, then Eq. (10) becomes

$$
\begin{aligned}
R_{n_{1} n_{1}^{\prime}} \simeq & \int d \boldsymbol{b} P_{e l}(b) \sum_{m_{1}^{\prime} m_{2}^{\prime} j_{2} l_{2} n_{2} m_{1} m_{2} \sigma^{\prime} \sigma^{\prime \prime}} \\
& \times \delta_{m_{1}-m_{1}^{\prime}, m_{2}-m_{2}^{\prime}}\left(l_{1} m_{1} s \sigma^{\prime} \mid j_{1} n_{1}\right)\left(l_{2} m_{2} s \sigma^{\prime} \mid j_{2} n_{2}\right) \\
& \times(-1)^{m_{2}^{\prime}-m_{2}}\left[Y_{l_{1} m_{1}}\left(\beta_{1}, 0\right) Y_{l_{1} m_{1}^{\prime}}^{*}\left(\beta_{1}, 0\right)\right]\left[Y_{l_{2} m_{2}}^{*}\left(\beta_{2}, 0\right)\right. \\
& \left.\times Y_{l_{2} m_{2}^{\prime}}\left(\beta_{2}, 0\right)\right]\left(l_{1} m_{1}^{\prime} s \sigma^{\prime \prime} \mid j_{1} n_{1}^{\prime}\right)\left(l_{2} m_{2}^{\prime} s \sigma^{\prime \prime} \mid j_{2} n_{2}\right) \\
& \times\left|K_{m_{1}-m_{2}}(\eta b)\right|^{2}\left|\mathcal{J}_{l_{2}}\right|^{2}
\end{aligned}
$$

and, as before, the $j_{2}$ sums can be performed to give

$$
\begin{aligned}
R_{n_{1} n_{1}^{\prime}} \simeq & \int d \boldsymbol{b} P_{e l}(b) \sum_{l_{2} m_{1} m_{2} \sigma^{\prime} \sigma^{\prime \prime}}\left(l_{1} m_{1} s \sigma^{\prime} \mid j_{1} n_{1}\right)\left(l_{1} m_{1} s \sigma^{\prime \prime} \mid j_{1} n_{1}^{\prime}\right) \\
& \times\left|\mathcal{J}_{l_{2}}\right|^{2}\left|K_{m_{1}-m_{2}}(\eta b)\right|^{2}\left|Y_{l_{1} m_{1}}\left(\beta_{1}, 0\right)\right|^{2}\left|Y_{l_{2} m_{2}}^{*}\left(\beta_{2}, 0\right)\right|^{2} .
\end{aligned}
$$

Upon substituting this in Eq. (9) and carrying out the angular momentum algebra, the numerator of $T_{k q}$ becomes

$$
\begin{aligned}
N_{k q} \simeq & \hat{k} W\left(l_{1} j_{1} l_{1} j_{1} ; s k\right) \int d \boldsymbol{b} P_{e l}(b) \sum_{l_{2} m_{1} m_{2}}\left|\mathcal{J}_{l_{2}}\right|^{2} \\
& \times\left|Y_{l_{2} m_{2}}^{*}\left(\beta_{2}, 0\right)\right|^{2}\left(l_{1} m_{1} k q \mid l_{1} m_{1}\right) \\
& \times\left|Y_{l_{1} m_{1}}\left(\beta_{1}, 0\right)\right|^{2}\left|K_{m_{1}-m_{2}}(\eta b)\right|^{2} .
\end{aligned}
$$

The denominator of $T_{k q}$ is also given by Eq. (14), but with $k=q=0$, being essentially the unpolarized cross section. The presence of Clebsch-Gordan coefficients involving only orbital angular momenta is a consequence of our (current) neglect of the neutron-target spin-orbit force.

Analyzing powers were derived previously within the TC model for transfer reactions between heavy nuclei [16]. There the Bessel function $K_{m_{1}-m_{2}}(\eta b)$ in Eq. (3) was replaced by the first term of its asymptotic expansion for large $\eta b$. An additional aim of this paper is to assess the accuracy of such an expansion to lowest order and also to higher orders, in the case of breakup studies of light nuclei.

\section{RESULTS FOR SPIN OBSERVABLES}

Two reactions are used to illustrate this work. The first is ${ }^{9} \mathrm{Be}\left({ }^{34} \mathrm{Si},{ }^{33} \mathrm{Si}\right) X$ at a beam energy of $70 \mathrm{MeV}$ per nucleon. This has been the subject of experiments carried out at the National Superconducting Cyclotron Laboratory (NSCL) at Michigan State University (MSU) [23] and has a neutron separation energy of $7.36 \mathrm{MeV}$. The second reaction, ${ }^{9} \mathrm{Be}\left({ }^{17} \mathrm{C},{ }^{16} \mathrm{C}\right) X$ at $60 \mathrm{MeV}$ per nucleon, has a ground state 
neutron separation energy of only $0.73 \mathrm{MeV}$. This knockout experiment has also been performed at the NSCL, but at a slightly different beam energy of $62 \mathrm{MeV}$ per nucleon [24]. We have chosen to calculate observables at $60 \mathrm{MeV}$, and to use the same two-body interaction descriptions as in Ref. [25], to compare our theoretical analyzing power results with those presented there. The ground state to ground state transitions in both cases are assumed to result from a $0 d_{3 / 2}$ neutron removal. With this $j_{1}=3 / 2$ projectile spin we expect a nonvanishing $T_{20}$ analyzing power [21].

\section{A. Model two-body interactions}

We use models for the neutron-target optical potential and core-target (ion-ion) interaction which are appropriate for intermediate-energy projectile beams. Following closely the procedures used in Ref. [15], the neutron-target optical potential is constructed by folding the ${ }^{9} \mathrm{Be}$ target point-nucleon matter density with the nuclear-matter effective nucleonnucleon $(N N)$ interaction of Jeukenne, Lejeune, and Mahaux (JLM) [26] and using the local density approximation. We assume a harmonic oscillator form for the ${ }^{9} \mathrm{Be}$ nucleon density. This microscopic approach to the complex nucleon optical potential provides very good agreement with measured reaction cross sections, and also elastic scattering angular distributions from light nuclear targets, over a range of incident nucleon energies, e.g., Ref. [27]. These include the incident energies of importance here. The JLM effective interaction parametrization itself extends over the entire energy range for which the neutron-target two-body $S$-matrix is required, thus our approach provides a consistent description of the nucleon-target interaction over the entire phase space probed by the TC reaction amplitude.

Since the TC model also assumes peripherality of the reaction, and that we are fully-external in the wave functions of the removed nucleon in the initial and final states, the coretarget interaction (absorption) enters only through $P_{e l}(b)$. This elastic channel survival probability for the core is instrumental in determining the active regions of impact parameter overlap of these wave functions and hence the resulting removal cross sections. We thus take proper account of the geometrical sizes of the core and target, and their associated strong absorption radius, by constructing the core-target interaction and $S$-matrices using the double-folding of their matter densities with an effective nucleon-nucleon $(N N)$ interaction. Details are given in Ref. [15].

The ${ }^{16} \mathrm{C}$ and ${ }^{33} \mathrm{Si}$-core point nucleon densities were assumed to have Gaussian form factors with range parameters chosen to reproduce the empirically deduced root mean squared (rms) matter radii. These were taken to be 2.70 and $3.16 \mathrm{fm}$, for ${ }^{16} \mathrm{C}$ and ${ }^{33} \mathrm{Si}$, respectively [28]. Extensive calculations for onenucleon knockout, including those for nucleon removal from tightly-bound states, show that the detailed radial form of these densities is unimportant for the calculated cross sections, see, e.g., Ref. [29]. The resulting $P_{e l}(b)$ has essentially a black-disk absorption profile and the cross sections are determined by the core and target matter rms radii, which then determine their strong absorption radius.
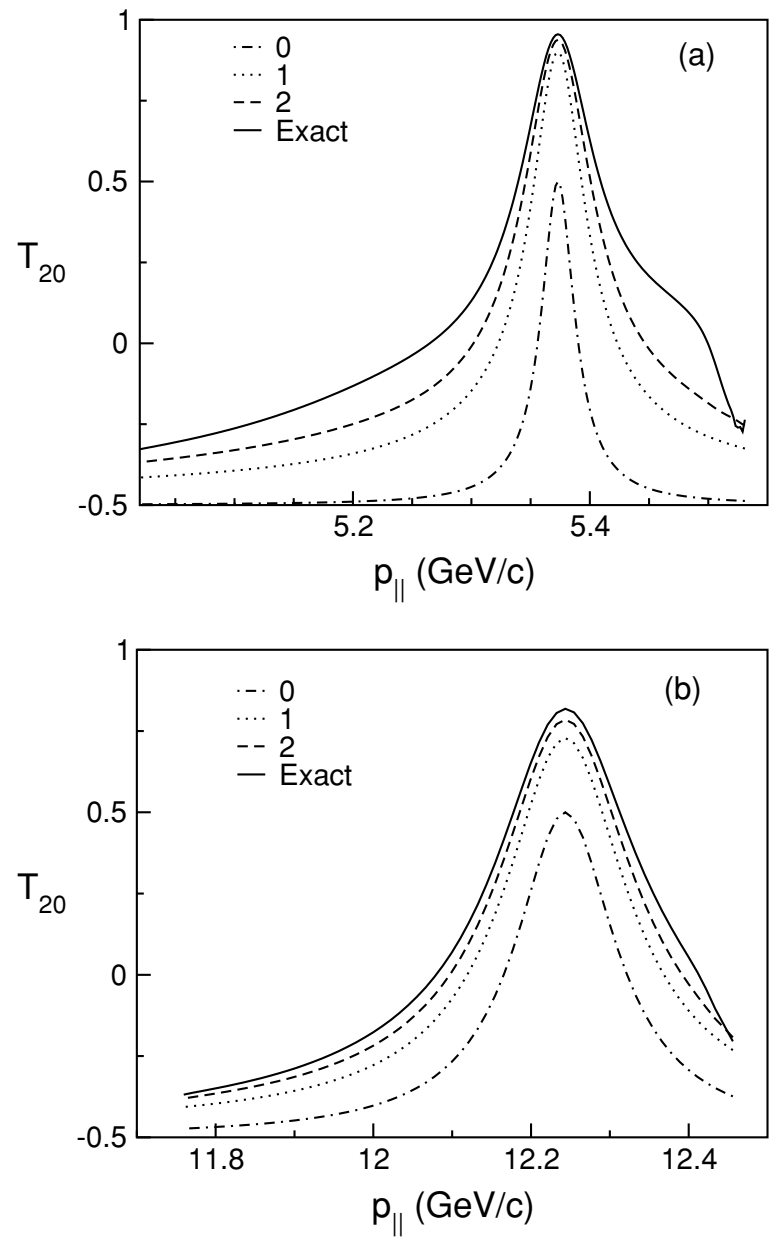

FIG. 1. $T_{20}$ as a function of the core parallel momentum for $d_{3 / 2}$ neutron removal from (a) ${ }^{17} \mathrm{C}$ and (b) ${ }^{34} \mathrm{Si}$ at 60 and $74 \mathrm{MeV}$ per nucleon, respectively. The numbers indicate the order in $\eta b$ used in the asymptotic expansion of the Bessel function.

\section{B. Analyzing power momentum distributions}

Calculations of $T_{20}$ as a function of the core parallel momentum in the laboratory, $p_{\|}$, are shown in Fig. 1. These calculations result from (i) the exact evaluation of the Bessel function in Eq. (11) (solid curves), and (ii) from truncation of the asymptotic expansion of the Bessel function to zeroth (dot-dashed), first (short-dashed), and second orders (longdashed); see Eq. (25) of Ref. [15] for the explicit form of this expansion. The analyzing powers have a positive peak in the region of the maximum in the calculated cross section. This peak is positioned at the value of core parallel momentum corresponding to $k_{1} \approx 0$. The $T_{20}$ decrease with increasing $\left|k_{1}\right|$, becoming negative. To understand this behavior, the polarized cross section momentum distributions must be examined. $T_{20}$ determines the fraction of the total cross section arising from each projectile angular momentum substate. For the initial $j_{1}=3 / 2$ states these polarized cross sections are [30]

$$
\begin{gathered}
\sigma_{3 / 2}=\sigma_{0}\left(1+T_{20}\right), \\
\sigma_{1 / 2}=\sigma_{0}\left(1-T_{20}\right),
\end{gathered}
$$



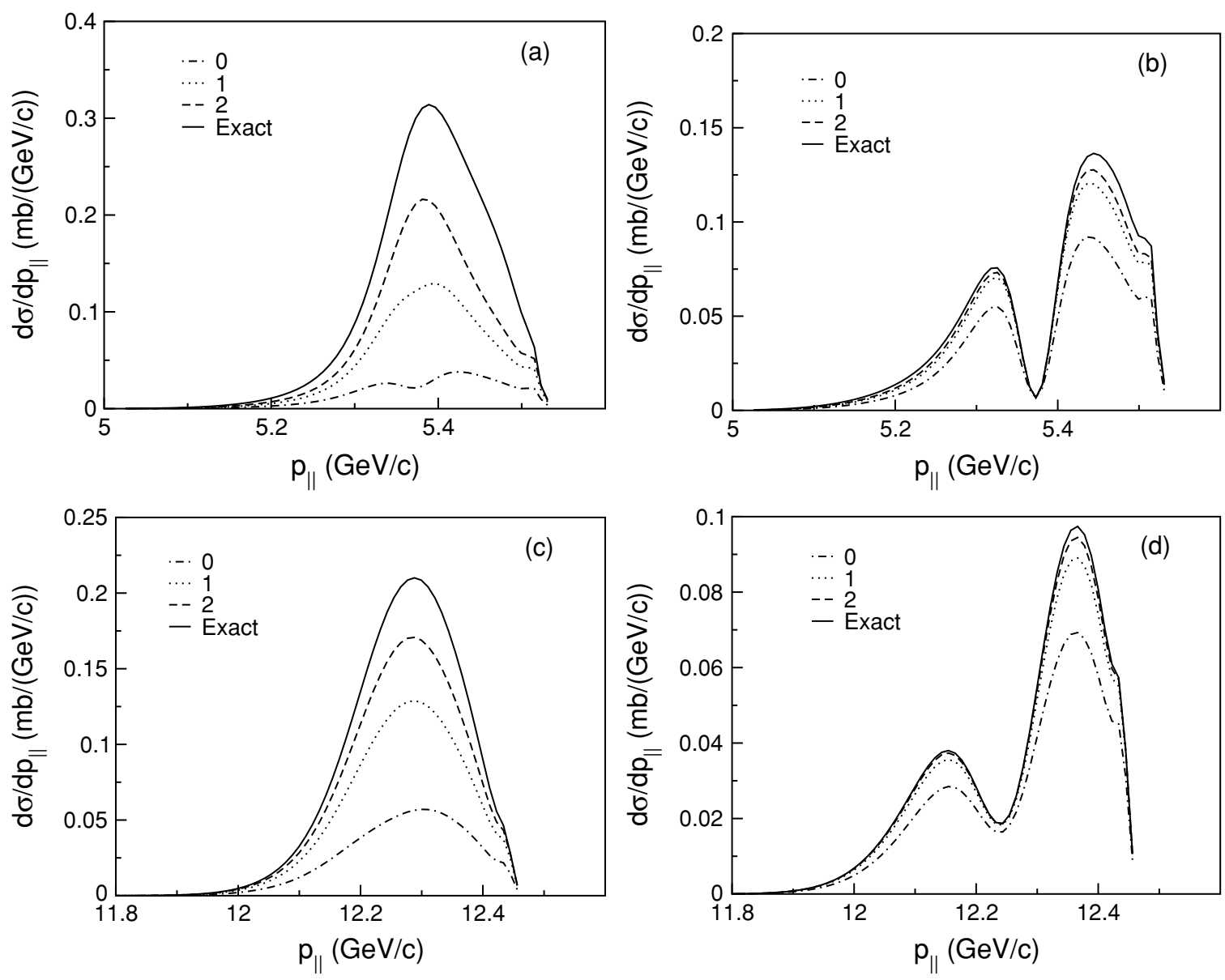

FIG. 2. Polarized cross sections (a) $\sigma_{3 / 2}$ for ${ }^{17} \mathrm{C}$, (b) $\sigma_{1 / 2}$ for ${ }^{17} \mathrm{C}$, (c) $\sigma_{3 / 2}$ for ${ }^{34} \mathrm{Si}$, and (d) $\sigma_{1 / 2}$ for ${ }^{34} \mathrm{Si}$, for different orders in the asymptotic expansion.

where the $\sigma_{\mu}$ denote the polarized cross sections for initial states with $n_{1}= \pm \mu$ and $\sigma_{0}$ is the cross section for an unpolarized beam. It is clear that $T_{20}>0$ favors a larger $\sigma_{3 / 2}$.

These polarized cross section results are displayed in Fig. 2. For both of our model systems we note that there is a large difference between $\sigma_{3 / 2}$ and $\sigma_{1 / 2}$ in the exact and in all orders of approximation of the Bessel function. The contribution from the more-aligned $n_{1}=3 / 2$ configuration is significantly bigger. The $\sigma_{1 / 2}$ are small at $k_{1}=0$ as they correspond to a situation where, classically, the neutron orbit is in a plane that contains the beam direction. This is sketched in Fig. 3. In this spin projection the wave function will contain only small components with $k_{1} \approx 0$. For $n_{1}=3 / 2$ on the other hand the angular momentum is aligned more nearly parallel to the beam direction, the neutron orbits in a perpendicular plane, and thus contributes more significantly to the cross section for small $k_{1}$. The situation is most clear when neglecting the neutron spin. In this limit the $m_{1}=0$ cross section vanishes identically at $k_{1}=0$, as has been shown in Ref. [31] within Glauber theory.

For each of our chosen systems, Fig. 2 also shows the convergence of the polarized cross section with different orders in the asymptotic expansion of the Bessel function. Their agreement is good for $\sigma_{1 / 2}$. For $\sigma_{3 / 2}$ however both the convergence and the agreement is very poor. It is clear that these approximations affect, almost exclusively, the most highly aligned configurations. This sensitivity is to be expected since the different orders in the expansion have an explicit $m_{1}$

(a)

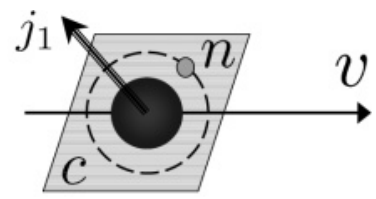

(b)

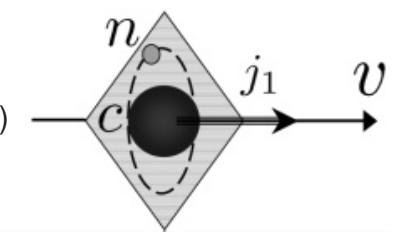

FIG. 3. Sketch of the state of rotation of the neutron $(n)$ around the core (c), with angular momentum $j_{1}$, from a classical point of view: (a) in a poorly aligned initial state, the plane that contains the orbit of the neutron contains also the beam velocity $v ;\left|n_{1}\right|$, the projection of $j_{1}$ onto $\boldsymbol{v}$, is small; (b) in a highly aligned initial state the plane of the orbit is perpendicular to the beam velocity; now $j_{1}$ and $v$ are parallel, thus the modulus of their projection onto one another, $\left|n_{1}\right|$, is big. 
TABLE I. Values of $T_{20}^{i}$ for one-neutron knockout from ${ }^{17} \mathrm{C}$ and ${ }^{34} \mathrm{Si}$ when neglecting (ns) and when including (so) the nucleon-target spin-orbit interaction. Calculations are obtained by exact evaluation and by truncation of the Bessel function to different orders in its asymptotic expansion.

\begin{tabular}{lrrrr}
\hline \hline Order & ${ }^{17} \mathrm{C}(\mathrm{ns})$ & ${ }^{34} \mathrm{Si}(\mathrm{ns})$ & ${ }^{17} \mathrm{C}(\mathrm{so})$ & ${ }^{34} \mathrm{Si}(\mathrm{so})$ \\
\hline Zeroth & -0.37 & -0.03 & -0.38 & -0.04 \\
First & 0.03 & 0.24 & 0.02 & 0.22 \\
Second & 0.21 & 0.33 & 0.20 & 0.32 \\
Third & 0.26 & 0.37 & 0.26 & 0.36 \\
Exact & 0.36 & 0.41 & 0.35 & 0.39 \\
\hline \hline
\end{tabular}

dependence, and hence the truncation effects are orientation dependent. We conclude that for the breakup of light nuclei, an exact treatment of the TC amplitude is crucial to obtaining reliable theoretical predictions for analyzing powers.

\section{Polarized total cross sections}

We now discuss the effect of beam polarization on the integrated breakup cross section. Relevant experiments are now those in which the final momenta of the fragments are not measured, and so are summed over. As was stated in Sec. II B, the $R_{n_{1} n_{1}^{\prime}}$ must now be integrated over all variables except the $n_{1}$ labels. This inclusive analyzing power is denoted here by $T_{20}^{i}$. $T_{20}^{i}$ is not the area under the curves in Fig. 1, but results from integrating separately the numerator and denominator of $T_{20}$. Table I presents the exact results obtained for ${ }^{17} \mathrm{C}$ and ${ }^{34} \mathrm{Si}$ and those from different orders of the Bessel function expansion.

We note that $T_{20}^{i}$ can reach a significant fraction of its maximum allowed value which, for $j_{1}=3 / 2$, is unity. $T_{20}$ is a measure of the sensitivity of the cross section to the beam polarization. Therefore, if there was significant (tensor) polarization of the secondary beam being used as the incident beam in the knockout reaction, significant differences in the theoretical predictions for integrated cross sections and of deduced spectroscopic factors might result. The sign of $T_{20}^{i}$ was also discussed in Ref. [25] with the use of geometrical arguments.

The different approximate predictions for $T_{20}^{i}$ are worse than was the case for the unpolarized cross section, as seen in the unpolarized momentum distributions of Ref. [15].

A calculation of the $T_{20}^{i}$ arising from the inelastic breakup or stripping mechanism was calculated using eikonal theory for the ${ }^{17} \mathrm{C}$ reaction [25]. This gave $T_{20}=0.23$. The stripping contribution from the TC model, the first term only in Eq. (6), gives 0.35. This suggests that the matching conditions inherent in the TC model predict a greater alignment effect. Experimental information is required to study and assess this effect quantitatively.

\section{Populations of the core substates}

In general the core nucleus has a nonzero intrinsic angular momentum $I_{c}$ and following the reaction some orientations of
$I_{c}$ will be favored. This can be predicted from the results of previous sections, where it was shown that the most highly aligned total angular momenta make the largest contributions to the cross section. Thus, with $\xi_{c}$ being the projection of $I_{c}$ on the $z$-axis, cross sections for large $\left|\xi_{c}\right|$ are expected to dominate. According to Eq. (3) each substate population $w\left(\xi_{c}\right)$ is then proportional to the probability

$$
\begin{aligned}
P\left(\xi_{c}\right)= & \int d \boldsymbol{b} P_{e l}(b) \sum_{j_{2} n_{2} l_{2} m_{2} n_{1} m_{1} m_{1}^{\prime} m_{2}^{\prime} \sigma^{\prime} \sigma^{\prime \prime} \xi_{n} \xi_{n}^{\prime}} \delta_{m_{1}-m_{1}^{\prime}, m_{2}-m_{2}^{\prime}} \\
& \times\left(l_{1} m_{1} s \sigma^{\prime} \mid I_{n} \xi_{n}\right)\left(l_{1} m_{1}^{\prime} s \sigma^{\prime \prime} \mid I_{n} \xi_{n}^{\prime}\right) \\
& \times\left(I_{n} \xi_{n} I_{c} \xi_{c} \mid j_{1} n_{1}\right)\left(I_{n} \xi_{n}^{\prime} I_{c} \xi_{c} \mid j_{1} n_{1}\right)\left(l_{2} m_{2} s \sigma^{\prime} \mid j_{2} n_{2}\right) \\
& \times\left(l_{2} m_{2}^{\prime} s \sigma^{\prime \prime} \mid j_{2} n_{2}\right)(-1)^{m_{2}^{\prime}-m_{2}}\left[Y_{l_{1} m_{1}}\left(\beta_{1}, 0\right)\right. \\
& \left.\times Y_{l_{1} m_{1}^{\prime}}^{*}\left(\beta_{1}, 0\right)\right]\left[Y_{l_{2} m_{2}}^{*}\left(\beta_{2}, 0\right) Y_{l_{2} m_{2}^{\prime}}\left(\beta_{2}, 0\right)\right] \\
& \times\left|K_{m_{1}-m_{2}}(\eta b)\right|^{2}\left|\mathcal{J}_{l_{2} j_{2}}\right|^{2} .
\end{aligned}
$$

When neglecting the neutron-target spin-orbit interaction, some of the sums can be carried out analytically, and $w\left(\xi_{c}\right)$ becomes

$$
\begin{aligned}
w\left(\xi_{c}\right)= & \int d \boldsymbol{b} P_{e l}(b) \sum_{l_{2} m_{1} m_{2}}\left|K_{m_{1}-m_{2}}(\eta b)\right|^{2}\left|\mathcal{J}_{l_{2}}\right|^{2}\left|Y_{l_{1} m_{1}}\left(\beta_{1}, 0\right)\right|^{2} \\
& \times\left|Y_{l_{2} m_{2}}^{*}\left(\beta_{2}, 0\right)\right|^{2} \sum_{k}(-1)^{k} \hat{k}^{2}\left(l_{1} m_{1} k 0 \mid l_{1} m_{1}\right) \\
& \times\left(I_{c} \xi_{c} k 0 \mid I_{c} \xi_{c}\right) W\left(l_{1} I_{n} l_{1} I_{n} ; s k\right) W\left(I_{c} I_{n} I_{c} I_{n} ; j_{1} k\right) .
\end{aligned}
$$

Calculations using the above are presented in Fig. 4 for the ${ }^{34} \mathrm{Si}$ reaction. The calculations, as a function of the core spin substate $\xi_{c}$, show the same features as those noted as a function of the neutron total angular momentum. There is a large difference between the contributions from $\left|\xi_{c}\right|=1 / 2$ and $\left|\xi_{c}\right|=3 / 2$, and a better agreement between the different approximate TC amplitudes for $\left|\xi_{c}\right|=1 / 2$.

\section{E. $\gamma$-ray angular distributions}

The ${ }^{33} \mathrm{Si}$ residual nucleus, with an assumed $I_{c}=3 / 2^{+}$ ground state, is known to have excited states at $1.01 \mathrm{MeV}$ and at $4.32 \mathrm{MeV}$ [32]. We have assumed that the stripped neutron originates from the $d_{3 / 2}$ valence shell of ${ }^{34} \mathrm{Si}$. Neutron removal from the $d_{5 / 2}$ shell is now considered, leaving the ${ }^{33} \mathrm{Si}$ nucleus in an excited $I_{c}=5 / 2^{+}$state, with decay to the ground state by $\gamma$-emission. The angular distribution of $\gamma$ radiation from this excited state decay is known to depend on the orientation of the angular momentum of the emitting object [33]. In the nuclear reactions under study here this information is provided by the populations of the different spin substates $\xi_{c}$, calculated in Sec. III D. Figure 5 shows the ${ }^{33} \mathrm{Si}\left(5 / 2^{+}, 4.32 \mathrm{MeV}\right)$ excited state substate populations [23]. These calculations use the exact Bessel function approach.

As expected, the most important populations are of the most aligned states. These populations are now used as input to the calculation of the $\gamma$-ray angular distributions [34,35]. The probability of emission of photons with wave vector $\boldsymbol{k}$, of 

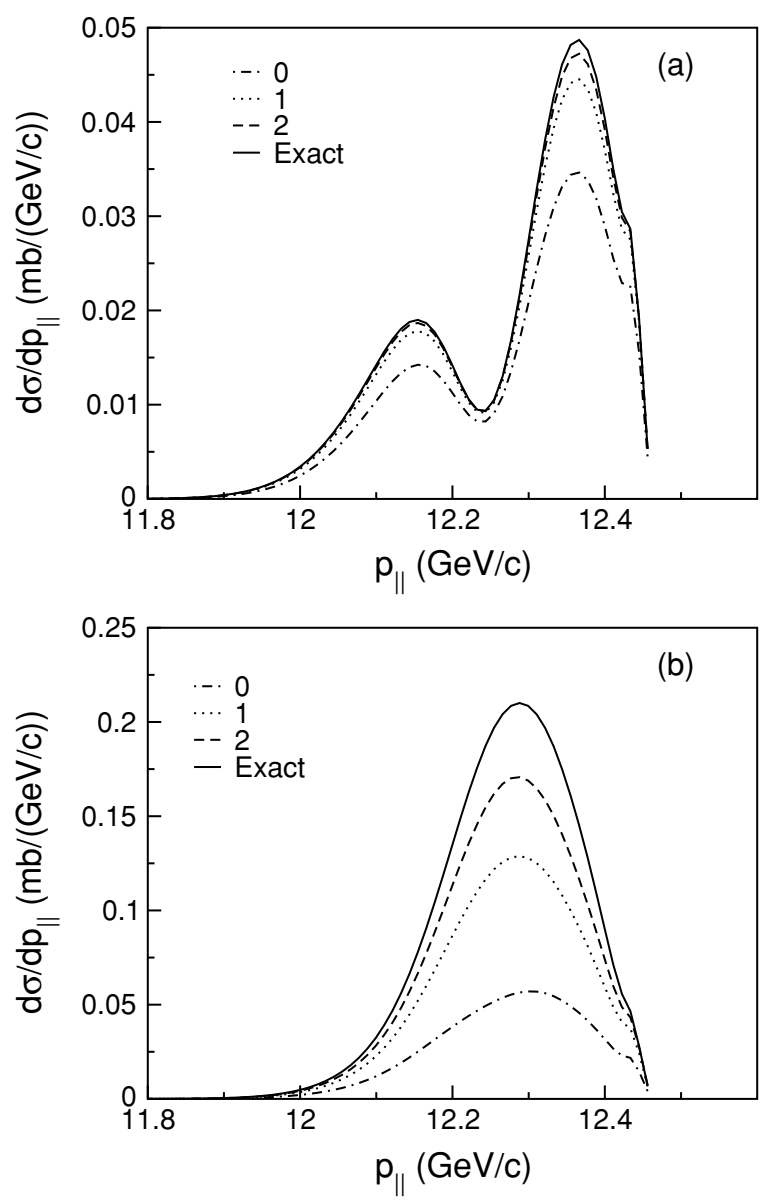

FIG. 4. Populations of the core ground state angular momentum substates, for several approximations, in the ${ }^{34} \mathrm{Si}$ reaction: (a) $\left|\xi_{c}\right|=$ $1 / 2$, (b) $\left|\xi_{c}\right|=3 / 2$.

any polarization, in the rest frame of the emitting body, is [33]

$$
\begin{aligned}
P(\boldsymbol{k})= & \frac{k}{2 \pi \hbar} \sum_{K L L^{\prime} \pi \pi^{\prime} q} B_{K}\left(I_{c}\right) P_{K}(\cos \theta)(-1)^{q+I_{c}-I_{0}+L^{\prime}-L-K} \hat{I}_{c} \\
& \times\left(L q L^{\prime}-q \mid K 0\right) W\left(I_{c} I_{c} L L^{\prime} ; K I_{0}\right) q^{\pi+\pi^{\prime}} \\
& \times\left\langle I_{c}\left\|T_{L}^{\pi}\right\| I_{0}\right\rangle\left\langle I_{c}\left\|T_{L^{\prime}}^{\pi^{\prime}}\right\| I_{0}\right\rangle^{*}
\end{aligned}
$$

where $I_{0}$ is the core ground state spin and

$$
B_{K}\left(I_{c}\right)=\sum_{\xi_{c}} w\left(\xi_{c}\right) \hat{K}\left(I_{c} \xi_{c} K 0 \mid I_{c} \xi_{c}\right) .
$$

The reduced matrix elements of the electromagnetic transition operators $\left\langle I_{c}\left\|T_{L}^{\pi}\right\| I_{0}\right\rangle$ and $\left\langle I_{c}\left\|T_{L^{\prime}}^{\pi^{\prime}}\right\| I_{0}\right\rangle$ are assumed here to be unity. If only a single transition $(E 2, M 1 \ldots)$ is considered, then this assumption does not affect the calculation of relative intensities as a function of angle. Here, a single multipole transition is assumed in each calculation. If all substates were equally populated then clearly the resulting radiation would be isotropic. Anisotropy is therefore a signal of the nonuniform distribution of the final core substate populations, i.e., of their alignment $[6,34]$. The areas under the curves in Fig. 5 give the $w\left(\xi_{c}\right)$ in Eq. (19).

The experimental momentum acceptance also plays a role in determining the expected $\gamma$-ray angular distribution. If only

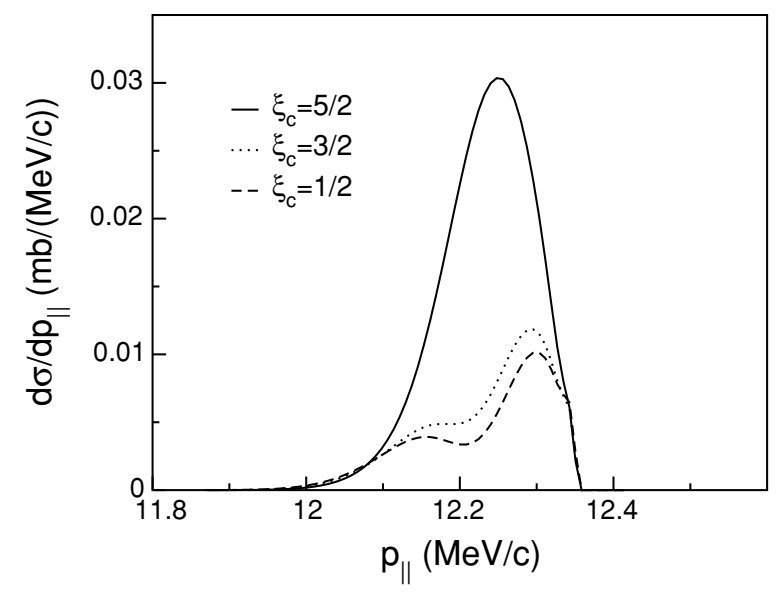

FIG. 5. Populations of the angular momentum substates with projections $\xi_{c}=1 / 2,3 / 2$, and $5 / 2$ of the ${ }^{33} \mathrm{Si}\left(5 / 2^{+}, E_{x}=4.32 \mathrm{MeV}\right)$ fragments.

a narrow momentum acceptance around the beam velocity is detected, then since in that region the difference between populations is greatest (Fig. 5), the radiation field will be most strongly anisotropic. A broader momentum acceptance of the core fragments, on the other hand, will lead to a more uniform distribution, as has been predicted in Ref. [2]. Figure 6 presents the angular distributions, calculated using the TC model, for three different values of the momentum acceptance. For assumed $E 2$ or $M 1$ transitions, our results concur with these expectations: the narrower the momentum bite, the larger the population differences, and the stronger the predicted $\gamma$-ray anisotropy.

\section{NUCLEON-TARGET SPIN-ORBIT INTERACTION}

To assess the importance of the spin-dependence of the nucleon-target two-body interaction responsible for breakup, we now supplement the JLM neutron-target potential with a realistic spin-orbit component. We adopt the spin-orbit potential form of Ref. [36],

$$
V_{\mathrm{so}}(r, E)=\frac{\hbar^{2}}{m_{\pi}^{2} c^{2}}\left[\lambda_{v}^{\mathrm{so}}(E)+i \lambda_{w}^{\mathrm{so}}(E)\right] U_{\mathrm{so}}(r) \boldsymbol{l} \cdot \boldsymbol{s},
$$

where $m_{\pi} c^{2}(=200 \mathrm{MeV})$ is the pion mass and $E$ is the nucleon incident energy. We take $U_{\text {so }}(r)$ to be

$$
U_{\mathrm{so}}(r)=\frac{1}{r} \frac{d \rho(r)}{d r}
$$

where $\rho(r)$ is the target matter density, as was used for the calculation of the JLM central potentials [37]. The parameters $\lambda_{v}^{\text {so }}(E)$ and $\lambda_{w}^{\text {so }}(E)$ are also taken from Ref. [36]. These are

$$
\begin{aligned}
& \lambda_{v}^{\text {so }}(E)=130 \exp (-0.013 E)+40, \\
& \lambda_{w}^{\text {so }}(E)=-0.2(E-20) .
\end{aligned}
$$

We first confirm that our neutron-target prescription is consistent with experiment by calculating the neutron elastic scattering analyzing power, $A_{y}$ and comparing with available 

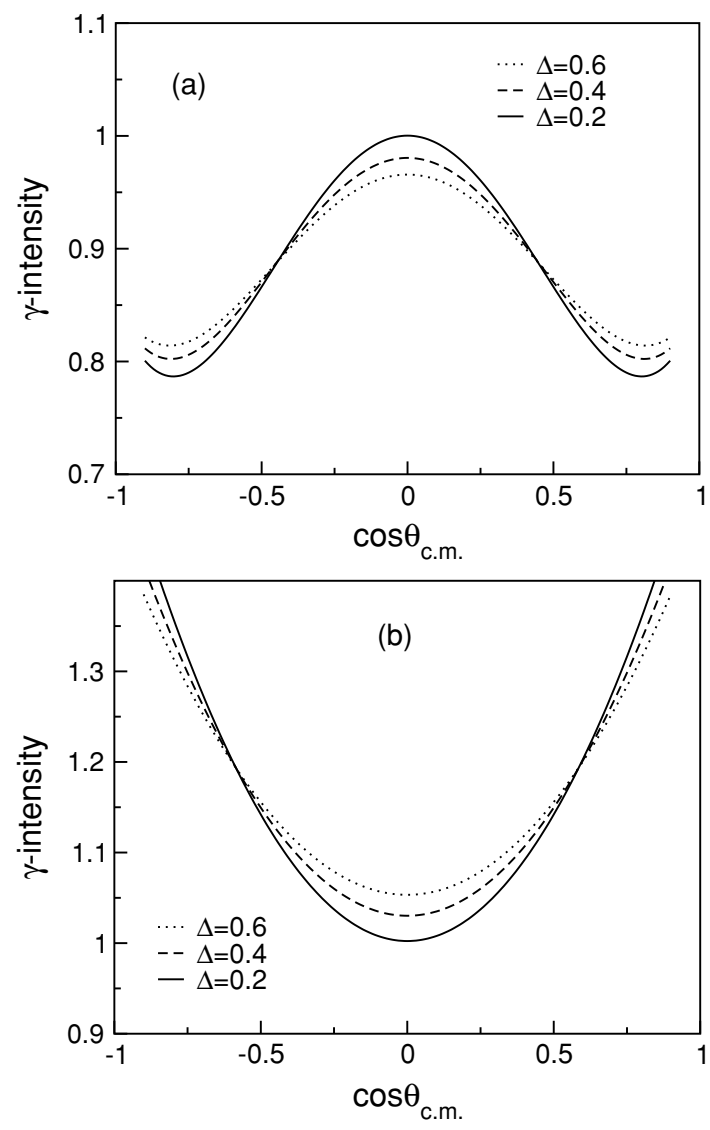

FIG. 6. $\gamma$-ray angular distribution from the ${ }^{33} \mathrm{Si}\left(5 / 2^{+}, E_{x}=\right.$ $4.32 \mathrm{MeV}$ ) state considering (a) $E 2$ and (b) $M 1$ transitions. $\theta_{\text {c.m. }}$. is the angle of the emitted radiation in the rest frame of the residue. The momentum acceptance $\Delta$ is given in $\mathrm{fm}^{-1}$ around $k_{1}=0,-\Delta \leqslant k_{1} \leqslant \Delta$. The intensities have been scaled to be 1 at zero angle.

data [38]. One such comparison is shown in Fig. 7 for neutron- ${ }^{9} \mathrm{Be}$ elastic scattering at $16.9 \mathrm{MeV}$. The agreement

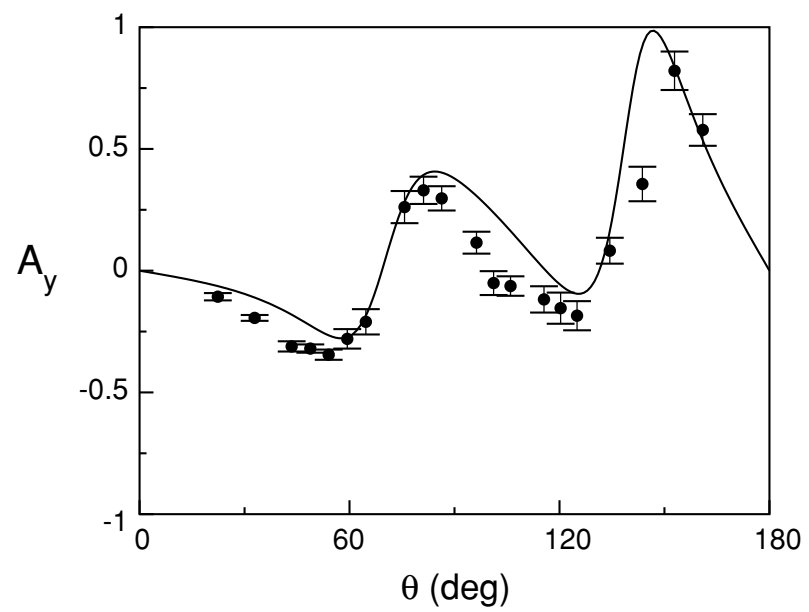

FIG. 7. Experimental results for $A_{y}$ in elastic scattering ${ }^{9} \mathrm{Be}(n, n){ }^{9} \mathrm{Be}$ [38], compared to our predictions, with an incident energy of $16.9 \mathrm{MeV}$.

\section{A. Cross section}

The breakup of ${ }^{34} \mathrm{Si}$ and ${ }^{17} \mathrm{C}$ are now revisited in the presence of spin-orbit distortion. Figures $8(a)$ and $8(b)$ show 


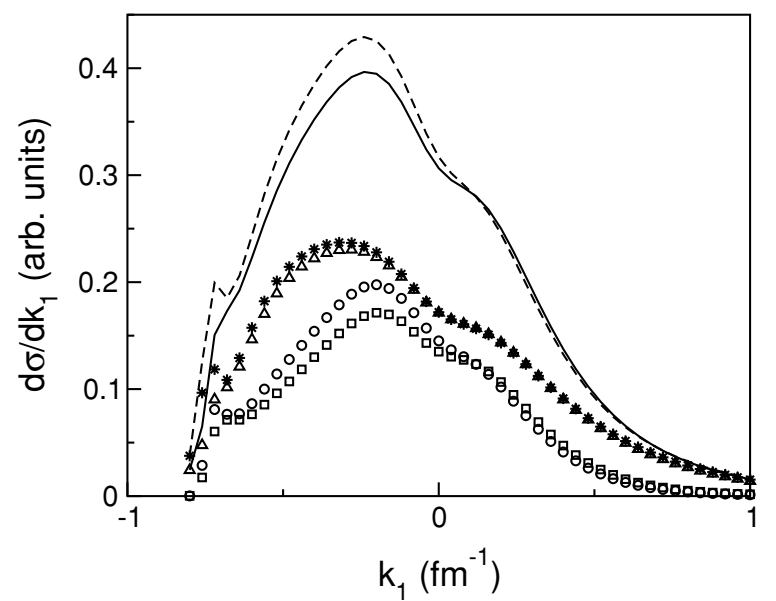

FIG. 9. Effects of nucleon-target spin-orbit distortion on the residue momentum distribution for the ${ }^{17} \mathrm{C}$ projectile. The calculation with (without) a spin-orbit interaction corresponds to the long-dashed (full) line. Stars (triangles) correspond to stripping with (without) spin-orbit, whereas circles (squares) represent elastic breakup with (without) spin-orbit.

the parallel momentum distribution of the ${ }^{16} \mathrm{C}$ and ${ }^{33} \mathrm{Si}$ ground state residues, respectively, calculated in the exact Bessel function approach. These are compared to the previous results of Eq. (5). The differences between the results of Eqs. (4) and (5) turns out to be small and within the experimental error bars on available integrated cross section measurements. The difference reaches its maximum at beam velocity and is sizable for negative $k_{1}$, corresponding to low neutron-target energies. The effects become smaller as the neutron-target relative energy is increased, due to the reduction in the strength of the spin-orbit interaction with incident energy, Eqs. (22).

Figure 9 displays the effect of spin-orbit distortion on the individual stripping and elastic breakup contributions to the momentum distribution of the ${ }^{16} \mathrm{C}$ residue. It is clear that the spin-orbit force induced differences are due to the elastic breakup rather than the stripping mechanism. This insensitivity in the stripping component is to be expected since the spin-orbit interaction is predominantly real, whereas the stripping cross section is dictated by the imaginary part of the nucleon-target phase shift, see, e.g., Ref. [20]. Since, at these energies, the knockout is dominated by the stripping mechanism, the spin-orbit distortion effects on the inclusive cross section and momentum distribution are therefore suppressed.

\section{B. Analyzing powers}

As was done in the previous section, for the cross section, we now investigate the effect of spin-orbit distortion on calculations of analyzing powers. This means we use Eq. (10) rather than Eq. (14). Figure 10 shows $T_{20}$ in the ${ }^{34} \mathrm{Si}$ case against the parallel momentum of the ${ }^{33} \mathrm{Si}$ cores, with and without the neutron-target spin-orbit interaction.

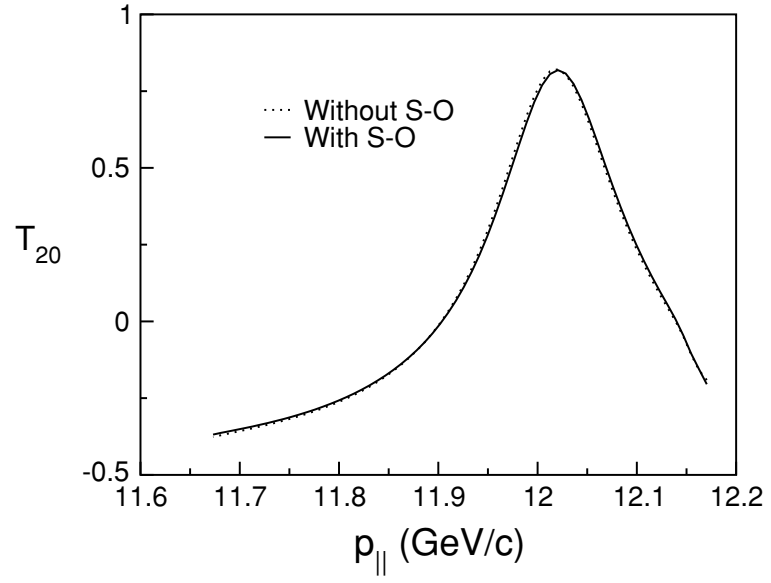

FIG. 10. Calculated $T_{20}$ versus the ${ }^{33} \mathrm{Si}$ residue parallel momentum, with and without neutron-target spin-orbit distortions, for a ${ }^{34} \mathrm{Si}$ projectile.

The similarity is quite remarkable and will induce a minimal effect on the polarized cross sections. Throughout this work everything points in the direction of the tensor analyzing power being an observable whose calculation can be carried out with a good degree of accuracy, without significant sensitivity to the different ingredients within the reaction model. In particular, the choices of the radius governing the core-target interaction and the neutron-target optical potential are of little importance. To add one more point to this argument, the inclusive analyzing power $T_{20}^{i}$ has been calculated when including the spin-orbit distortion. These results are also shown in Table I. They are also very similar.

\section{CONCLUDING REMARKS}

Spin observables of one-nucleon knockout reactions, and the effects of the neutron-target spin-orbit interaction, have been considered using the transfer-to-the-continuum direct reaction formalism. The theoretical framework for the calculation of analyzing powers is presented, and some test cases are studied. Our results show that the reaction analyzing powers can be large and may reach a significant fraction of their maximum allowed values. We show that the calculated analyzing powers are very sensitive to the use of approximations to the TC model amplitude and hence that a proper (exact) treatment of this transfer amplitude is required for accurate calculations of analyzing powers.

The spin-dependent formalism developed also enables predictions of the angular distributions of $\gamma$-rays emitted from excited states of the heavy reaction residues. The potential of such measurements as an additional source of structure information have been discussed in the literature [6,34]. We find significant, final-state-dependent, angular dependence in these calculated angular distributions and which are sensitive to the momentum acceptance of the recoiling residues.

Finally, we have assessed the effects of neutron-target spin-orbit distortion on both the unpolarized knockout 
reaction cross sections and their associated analyzing powers. This interaction has been neglected in analogous eikonal reaction model treatments. The model interaction chosen was consistent with measurements of the analyzing power in the $n+{ }^{9} \mathrm{Be}$ binary system. We find that the effects of the nucleon-target spin-orbit distortions are small, suggesting that, for the systems, energies, and polarization observables considered here, the formal and numerical complications of this spin-dependence can safely be neglected in such knockout calculations.

\section{ACKNOWLEDGMENT}

This work was supported by the United Kingdom Engineering and Physical Sciences Research Council (EPSRC) under Grant No. GR/M82141.
[1] P. G. Hansen and B. M. Sherrill, Nucl. Phys. A693, 133 (2001).

[2] P. G. Hansen and J. A. Tostevin, Annu. Rev. Nucl. Part. Sci. 53, 219 (2003).

[3] D. Cortina-Gil et al., Phys. Lett. B529, 36 (2002).

[4] E. Sauvan et al., Phys. Lett. B491, 1 (2000).

[5] A. Bonaccorso and D. M. Brink, Phys. Rev. C 38, 1776 (1988).

[6] H. Olliver, T. Glasmacher, and A. E. Stuchbery, Phys. Rev C 68, 044312 (2003).

[7] H. Okuno et al., Phys. Lett. B335, 29 (1994).

[8] K. Matsuta et al., Phys. Lett. B281, 214 (1992).

[9] K. Asahi et al., Phys. Lett. B251, 488 (1990).

[10] J. S. Al-Khalili and R. C. Johnson, Nucl. Phys. A546, 622 (1992).

[11] R. J. Glauber, Lectures in Theoretical Physics (Interscience, New York, 1959), p. 315.

[12] H. Hashim and D. M. Brink, Nucl. Phys. A476, 107 (1988).

[13] A. Bonaccorso, D. M. Brink, and L. Lo Monaco, J. Phys. G 13, 1407 (1987).

[14] A. Bonaccorso and D. M. Brink, Phys. Rev. C 44, 1559 (1991).

[15] A. García-Camacho, R. C. Johnson, and J. A. Tostevin, Phys. Rev. C 71, 044606 (2005).

[16] J. Gómez-Camacho, Nucl. Phys. A496, 403 (1989).

[17] L. Lo Monaco and D. M. Brink, J. Phys. G 11, 935 (1985).

[18] H. Hasan and D. M. Brink, J. Phys. G 4, 1573 (1978).

[19] F. Stancu and D. M. Brink, Phys. Rev. C 32, 1937 (1985).

[20] A. Bonaccorso, Phys. Rev. C 60, 054604 (1999).

[21] J. Gómez-Camacho and R. C. Johnson, Polarization in Nuclear Reactions, Chap. 3.1.5 of Scattering (Academic Press, London and San Diego, 2002), p. 1414.

[22] D. A. Varshalovich, A. N. Moskalev, and V. K. Khersonskii, Quantum Theory of Angular Momentum (World Scientific, Singapore, 1988).
[23] J. Enders et al., Phys. Rev. C 65, 034318 (2002).

[24] V. Maddalena et al., Phys. Rev. C 63, 024613 (2001).

[25] R. C. Johnson and J. A. Tostevin, in Analysing power of neutron removal reactions with beams of neutron-rich nuclei, edited by H. Yabu, T. Suzuki, and H. Toki, Spins in Nuclear and Hadronic Reactions, Proceedings of the RCNP-TMU Symposium, Tokyo, Japan, 26-28 October 1999 (World Scientific, Singapore, 2000), p. 155.

[26] J. P. Jeukenne, A. Lejeune, and C. Mahaux, Phys. Rev. C 16, 80 (1977).

[27] J. S. Petler, M. S. Islam, R. W. Finlay, and F. S. Dietrich, Phys. Rev. C 32, 673 (1985).

[28] A. Ozawa et al., Nucl. Phys. A693, 32 (2001).

[29] A. Gade et al., Phys. Rev. Lett. 93, 042501 (2004).

[30] R. C. Johnson, Nuclear reactions with polarized lithium and deuteron beams, edited by H. Ogata, M. Fujiwara, and A. Shimizu, Lecture Notes of the 1983 RCNP Kikuchi Summer School, Kyoto, Research Center for Nuclear Physics, Osaka (1983), p. 193.

[31] H. Esbensen, Phys. Rev. C 53, 2007 (1996).

[32] P. M. Endt et al., Nucl. Phys. A633, 1 (1998).

[33] H. J. Rose and D. M. Brink, Rev. Mod. Phys. 39, 306 (1967).

[34] A. E. Stuchbery, Nucl. Phys. A723, 69 (2003).

[35] M. E. Rose, Elementary theory of angular momentum (Wiley \& Sons, New York, 1957).

[36] E. Bauge, J. P. Delaroche, and M. Girod, Phys. Rev. C 58, 1118 (1998).

[37] C. W. De Jager et al., At. Data Nucl. Data Tables 14, 479 (1974).

[38] R. C. Byrd et al., Nucl. Phys. A427, 36 (1984). 